\title{
Analysis of power lines span geometry based on TLS measurements
}

\author{
Andrzej Kwinta ${ }^{1, *}$, Karolina Ważydrąg ${ }^{1}$, and Mariusz Zygmunt ${ }^{1}$ \\ ${ }^{1}$ University of Agriculture in Krakow, Faculty of Environmental Engineering and Land Surveying, 21 A. Mickiewicz Av., \\ 31-120 Krakow, Poland
}

\begin{abstract}
The inventory measurements of utility infrastructure are crucial issues in the field of engineering geodesy. Secure usage of power lines requires to monitor their geometry. According to classical approach to measurements of geometry of overhead power lines, it usually allows only to measure directly. Geodetic methods cannot be applied due to measuring limitations (lack of an appropriate area to conduct a measurement). Currently in engineering geodesy it is becoming more common to use methods of laser scanning as well as Unmanned Aerial Photogrammetric Survey. In the Paper, the procedure of determining of an overhead power line's geometry is presented on the example of an one span measured with Terrestrial Laser Scanning methods. Field measurements were conducted by using laser scanner. Afterwards the point cloud was processed in order to receive the geometry of conductor. Finally, results were presented is tabular and graphic form.
\end{abstract}

\section{Introduction}

The existence of societies in the strongly urbanized and industrialized areas is based on the usage of technical infrastructure. In modern times it is impossible for the economy as well as the society to operate without various media delivered with the (dependable) technical infrastructure [1]. It can be told that the area is entangled with overhead, terrestrial and underground networks of utility infrastructure. The proper operation of those infrastructure is significant for well being of societies.

Constructions of technical infrastructure are crucial elements of human living space [2]. Those constructions determine the comfort and the security of men existence. People get used to benefits that are provided by media delivered with utility infrastructure. A present-day city inhabitant do not imagine the existence without access to various media. Therefore, it is important to control the condition of utility infrastructure form the operational as well as the geometrical point of view.

Power lines transmit electrical energy from generating sites to power consumers [3]. Usually (due to harmfulness of radiation) overhead power lines are being constructed in undeveloped areas, very often such as woodlands. In those situations capabilities of conducting measurements by using classical geodetic methods are limited and the only applicable way of measurement is a direct one or a one with usage of remote sensing methods. Lately ALS methods and low-altitude UAV photogrammetry are becoming more commonly used to conduct power lines' measurements.
The implementation of GNSS measuring systems as well as the development of laser scanning technologies undoubtedly facilitate the conducting of geodetic measurements. In the Paper is presented the issues of determining a power line's span geometry based on laser scanning measurement. Therefore, the method of performing TLS measurements and calculating its results is not the subject of this work. Measurement TLS has been described only to a minimal extent. Presented issues are illustrated with the example. The example uses measured and processed data from TLS measurements in the form of a point cloud (point coordinates).

\section{The geometry of overhead power line}

An overhead power line consists of many elements, which undergo measurements and analyses [3]. The power line is an element of the infrastructure, which transmits electrical energy from a generating site to end consumers (users). A single power line can be divided into spans, which are sections of cable between two hook points. According to parameters of a power line, it can be constructed differently and a line's geometry can differ significantly.

Single conductor between two support points can be considered as freely hanging rope, which the weight has an effect on. In such an instance the cable takes shape of a catenary. The freely hanging cable can be also described with a quadric polynomial. Below in the picture 1, basic geometric parameters of single cable are presented.

\footnotetext{
* Corresponding author: andrzej.kwinta@ur.krakow.pl
} 


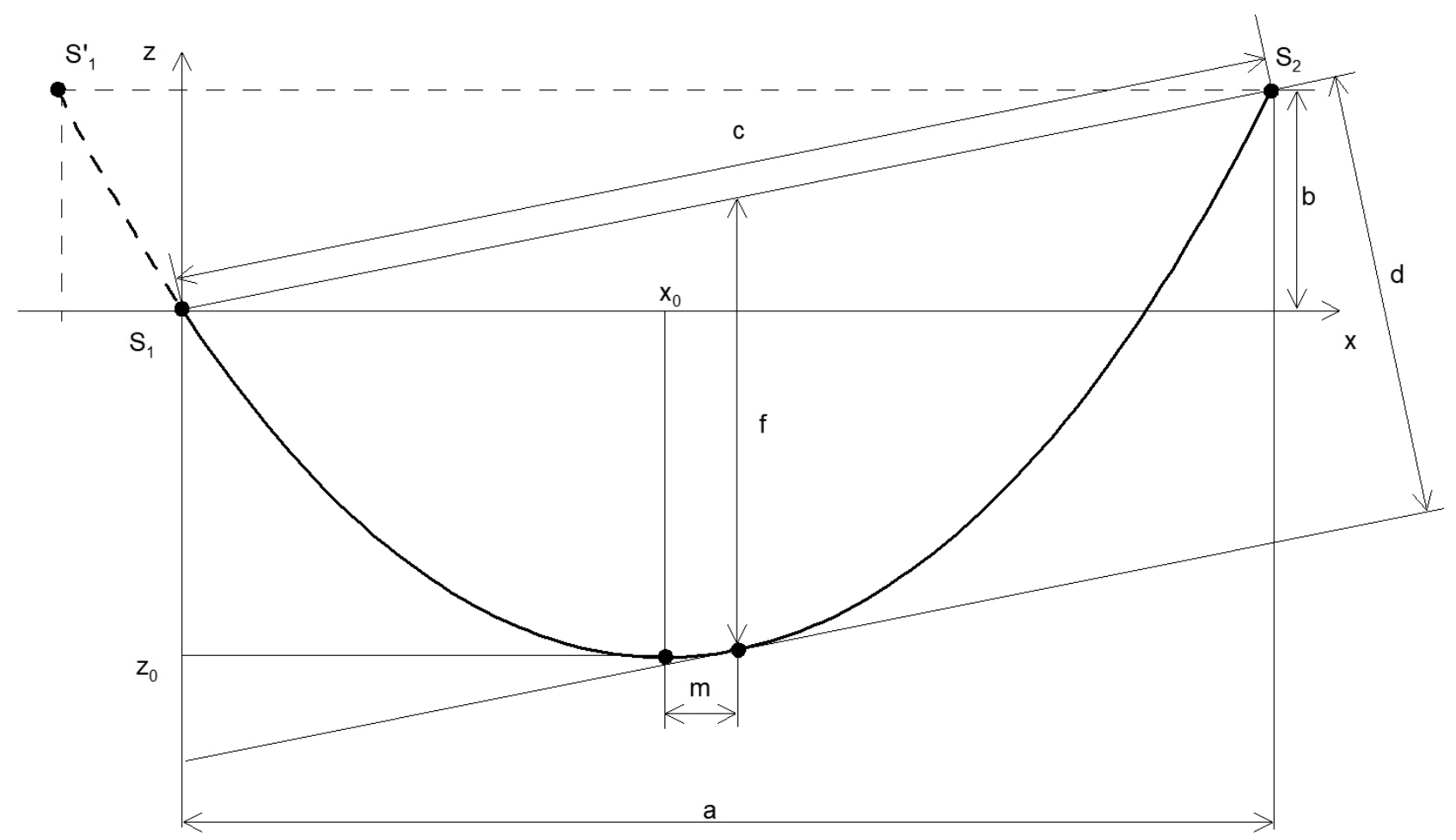

Fig. 1. Geometric parameters of span of freely hanging cable.

According to the Fig. 1 following geometric parameters can be distinguished:

- $\quad$ span length $(a)$ - a horizontal distance between axes of adjacent supporting structures (cable support points)

- chord $(c)$ - a straight line segment, whose endpoints are cable support points

- sag $(f)$ - the biggest vertical distance between a cable and a chord

- slope (b) - a vertical distance between two support points of one cable of an inclined span

- eccentricity $(m)$ - of an inclined span; a distance between the lowest point of a cable and a centre of a span

- devilation $(d)$ - the biggest distance between a curve of hanging cable and a chord; measured perpendicularly to a chord

- length $(\alpha)$ - a length of a cable between two insulators

- the lowest point $\left(X_{0}, Z_{0}\right)$ - coordinates of the lowest point of a cable

There are two theoretical equations which describes a cable geometry $[4,5]$ :

- catenary

$$
z(x)=k \cdot \cosh \left(\frac{x-x_{0}}{k}\right)+S
$$

where:

$k$ is a catenary shape parameter $x_{0}-$ a coordinate of the lowest point $S-$ a translation in vertical plane
- parabola

$$
z(x)=p x^{2}+q x+r
$$

where $p, q, r$ are parameters of parabola $(q=0$ when a parabola is a polynomial approximation of a catenary).

On the basis of abovementioned formulas and the Fig. 1, following equations for geometric parameters of single cable can be formulated according to adopted theoretical function:

- span length

$$
a=x_{\max }-x_{\min }
$$

$-\quad$ slope

$$
b=\left|z\left(x_{\max }\right)-z\left(x_{\min }\right)\right|
$$

- chord

$$
c=\sqrt{a^{2}+b^{2}}
$$

- sag

$$
f=k\left[\frac{b g}{a}-\cosh (g)\right]+\frac{b x_{0}}{a}-S
$$

where $g=\operatorname{arcsinh}(b / a)$

- parabola

$$
f=\frac{(b-a q)^{2}}{4 a^{2} p}-r
$$




$$
\begin{aligned}
& \text { - eccentricity } \\
& \qquad \text { catenary } \\
& \qquad m=k \cdot \operatorname{arcsinh}\left(\frac{b}{a}\right)
\end{aligned}
$$

- parabola

$$
m=\frac{b}{2 a p}
$$

- devilation

- catenary

$$
d=\frac{a \cdot f}{c}
$$

- parabola

$$
d=\frac{(b-a q)^{2}-4 a^{2} p r}{4 a p \sqrt{a^{2}+b^{2}}}
$$

- length

$$
\begin{gathered}
\alpha=k\left[\sinh \left(\frac{a-x_{0}}{k}\right)-\sinh \left(\frac{-x_{0}}{k}\right)\right] \\
\alpha=\frac{t \sqrt{t^{2}+1}+\operatorname{arcsinh}(t)-q \sqrt{q^{2}+1}-\operatorname{arcsinh}(q)}{4 p}
\end{gathered}
$$

where $t=2 a p+q$

- the lowest point

- catenary

$$
\begin{gathered}
X_{0}=x_{0} \\
Z_{0}=k+S
\end{gathered}
$$

- parabola

$$
\begin{gathered}
X_{0}=-\frac{q}{2 p} \\
Z_{0}=\frac{4 p r-q^{2}}{4 p}
\end{gathered}
$$

As it can be seen both equations (1) and (2) have three parameters, that is why to unambiguously determine equations, three points of measurement of a cable are needed. Taking into consideration span's geometric parameters, two opposite support points of a conductor and one point on a conductor has to be measured.

\section{Methods of measurement}

Measurements of overhead power lines are, as a rule, conducted with geodetic methods. Not long time ago basic and most popular method was measuring of vertical and horizontal angles as well as distances. Currently methods based on low-altitude photogrammetry and mobile scanning systems (aerial as well as automotive). In some cases (a measurement of deflection only) other non-geodetic (direct) measurement methods, which requires climbing up on supporting structures, are being used.

Regarding direct measuring methods, following are being used $[6,7]$ :

- driven oscillations method (a cable oscillation is driven with shudder and a time is measured),

- surveying targets method (a measurement of an vertical angle to cable's tangent),

- levelling rods method (outlining a tangent in the point of maximal sag).

Following geodetic methods are being used [8-12]:

- angular intersection (conducted in vertical plane),

- trigonometric method (a measurement of vertical and horizontal angles as well as distances),

- aerial photogrammetric survey (classical and lowaltitude UAV),

- laser scanning (aerial, mobile, terrestrial),

- remote sensing methods,

- GPS measurement.

Each of methods enlisted above has its advantages and disadvantages, what determine the choice of most suitable measurement method by taking into consideration actual needs, measurement's conditions as well as a geometry of measured object.

Laser scanning technology, which a few years ago was considered to be the most innovative and modern one, currently has become the primary measurement method used in geodesy. A common access to measuring instruments as well as computer software has led to ubiquitous application of this method to measuring any of engineering objects. One of basic parameters connected with conducting a measurement with laser scanning method is choice of appropriate measurement points' density in accordance with an object's geometry as well as requirements to a measurement accuracy. Too low density of points causes a decline in accuracy of determining a geometry of object, but in the same time this leads to speeding up measurements and calculations. On the other hand, too high density of points causes significant extension of measurement time and leads to difficulties in reprocessing obtained data. A reprocessing of measurement outcomes requires performing some operations, which are dependent on an available computer software.

\section{Data evaluation}

As a result of the laser scanning method measurements, one or more point clouds are obtained. Those point clouds have to be reprocessed in order to calculate geometrical elements of a measured object. Certain operations have to be performed to extract measured points of measured object from a point cloud and afterwards calculate object's geometrical elements [1319]. 
Producers of measuring instruments and computer software are delivering solutions for reprocessing data in automatic or semi-automatic form [20], but for this Paper the manual reprocessing of outcomes has been conducted.

In order to obtain geometric data on the basis of the laser scanning method measurement, the following calculation steps have to be taken:

1. Downloading measurement data from measuring instrument to computer

2. Merging of point clouds (if there are more than one) and registration of data in a presupposed spherical coordinate system

3. Separating the object from point cloud, extraction of single cables in the analysed span of power line

4. Transforming the measuring three-dimensional coordinate system into two-dimensional coordinate system connected with the certain cable in the certain span of the power line (the cable's hook point at the origin of coordinate system, the axis of coordinate system in the same vertical plane with the cable's axis of geometry)

5. Estimated determining of the cable's theoretical equation (e.g. a catenary or a parabola)

6. Direct determining of parameters of the cable's theoretical equation in accordance with the Least Squares method (the least-squares function approximation)

7. Calculating conductor's parameters (vide Part 2 of the Paper)

8. Analysing the accuracy (determining of errors of calculated equations' parameters and geometric parameters)

9. Transforming results into spherical coordinates system

10. Presenting results of measurements and calculations (in tabular and graphic forms)

Abovementioned steps connected with calculations are automated to some bigger or smaller extent depending on a computer software used for reprocessing, but the verification of calculations' correctness is necessary by the operator. Authors of the Paper want to clearly state that the automation of calculations is desired, but on the other hand it is essential to verify outcomes of particular calculation stages. This publication is mainly about steps $5-10$ of the presented procedure. The authors assumed that steps 1-4 of the procedure cover issues that will be the subject of separate publication.

Taking into account the density of scanning, and therefore the volume of data (size of a point cloud), conducting calculations might require high-speed computers and hard discs of high capacity and moreover calculations might be highly time-consuming. In the next section of the Paper, the process of conducting calculations of the measured power line's span is described.

\section{Example}

The measurement presented in the Paper were conducted for scientific purposes, that is why the high density of measurement points has been obtained [21]. This set of data has been collected for the needs of various analyses. In the Paper only the part of data has been used.

The measurement has been conducted with the scanner Leica Scan Station P30 [22] in KrakowMydlniki (Fig. 2). The measured object was a span of $220 \mathrm{kV}$ power line of 300 meters range. The power line consists of six working conductors and two ground wires. In the Fig. 2, the fragment of measured power line is presented.

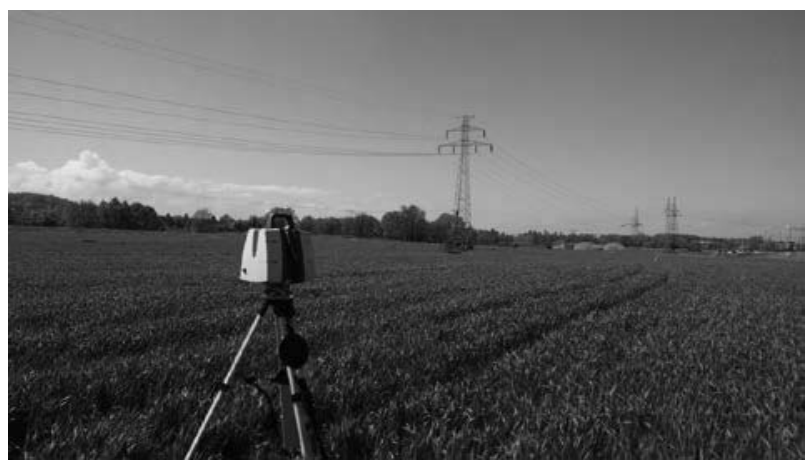

Fig. 2. The fragment of the measured power line and the set measuring instrument.

Measurements were conducted on three positions with the use of six adjustment points. Adjustment points were measured classically and their coordinates were marked out in the local coordinates system. The single working cable (marked with red in the Fig. 3) was chosen for analysis, which the point cloud of 903516 points was selected for, what gives the average density of 3 points per millimetre of the cable.

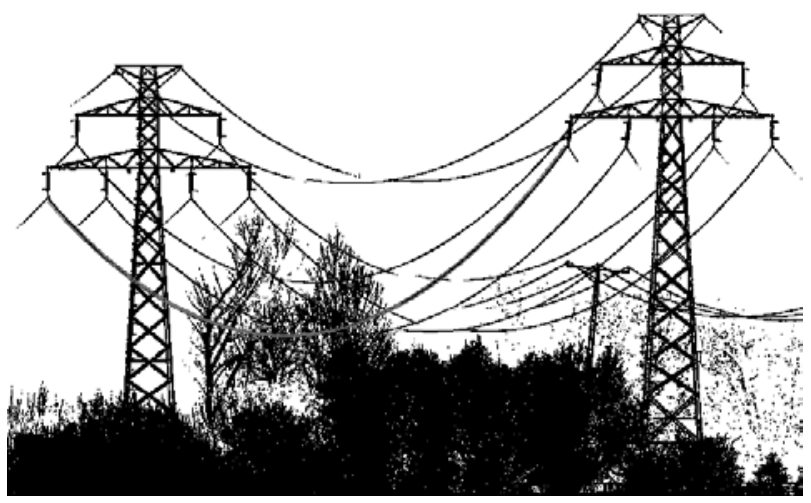

Fig. 3. Visualisation of the point cloud with the analysed cable marked in red colour.

The data from the measuring instrument was downloaded and merged with LEICA CYCLONE software [23]. Particular point clouds from different measurement positions were combined into one set and saved as '*.pod' text file.

In order to analyse outcomes, the separation of data was needed and the point cloud had to be divided to 
extract particular cables, what allowed to determine their geometric parameters. This reprocessing was conducted with Bentley DESCARTES software [24]. In the Fig. 3 the single cable of the analysed power line's span picked for the further analysis is marked (in red colour). Only one single cable was chosen due to the fact that the analysis of all cables did not give any additional information in this particular case. It is crucial during choosing data to correctly identify hook points of the cable on the supporting structure, because this in fact determines span's geometric parameters.

To conduct the calculation the original software has been created in DELPHI programming language in RAD Studio application [25]. Data transformations, estimated calculations, precise calculations as well as determination of approximation's errors were done with this software. Firstly, as results of conducted calculations theoretical equations of curves approximating the electric cable were determined by calculating estimated values and later precise ones of equations' parameters:

- the function of a catenary

$$
z=1136.296 \cosh \left(\frac{x-136.290}{1136.296}\right)-1144.573
$$

- the function of a parabola

$$
z=0.0004414 x^{2}-0.120381 x-0.076
$$

On the basis of determined theoretical equations and formulas (3)-(11) presented in part 2 of the Paper the span's parameters have been calculated and outcomes are compiled below in the Table 1.

While analysing outcomes compiled in the Table 1 it had to be stated that values of geometric parameters of cable's span received with the approximation theory were very similar for either the catenary as well as the parabola. The maximum difference equalled around 0.1 $\mathrm{m}$ and concerned the eccentricity, what was caused by the distinct location of the lowest point (difference of $0.073 \mathrm{~m}$ ) according to both of theories. Regarding the sag, the difference was of $0.002 \mathrm{~m}$, what was the negligible value, while taking into account the total value of the sag.
Along with the approximation of outcomes of span's measurements, not only theoretical functions (12) and (13) were calculated, but average errors of approximation were determined. For the function of a catenary the average error of approximation equalled $0.035 \mathrm{~m}$, while for the function of a parabola the average error equalled $0.039 \mathrm{~m}$. When comparing received outcomes it had to stated that both values were similar and both theoretical functions described the measured power line's cable correctly.

Numerical results of calculations were saved as appropriate text files, which were used to create the visualisation in a graphic program. The Bentley MICROSTATION software [26] was used to create the data visualisation. The point cloud obtained from measurements uploaded to the program as the '*.pod' text file (default layer of picture) and later appropriate calculations' results were placed on the next layers of the picture. During calculations the proper textual structure of files was conferred and therefore calculations' results in loadable form were possible to have been included on layers of the picture.

For each of theoretical curves (the catenary, the parabola) following layers of the picture were created:

- the layer of the theoretical curve - contain the equation in textual form and the theoretical curve marked with appropriate colour;

- the layer of cable's parameters - containing the numerical sheet of geometric parameters of the cable's span and graphic form of appropriate points as well as line segments in the picture;

- the layer of the errors' analysis - containing textual description of cable's approximation errors for particular points marked in different colours. The division of errors into 10 categories (every $7 \mathrm{~mm}$ ) was adopted and to each category different colour was assigned (Fig. 4).

As the example of results presented in graphic form, in the picture below the snapshot of the default layer and the layer of the errors' analysis for the cable selected for calculations. Also, the small part of the analysed cable is magnified (Fig. 4).

Table 1. Geometric parameters of cable's span (all results are presented in meters).

\begin{tabular}{|l|c|c|c|}
\hline Parameter & Catenary & Parabola & Difference \\
\hline Span length & 298.019 & 298.019 & 0.000 \\
\hline Slope & 3.346 & 3.327 & 0.018 \\
\hline Chord & 298.037 & 298.037 & 0.000 \\
\hline Sag & 9.879 & 9.877 & 0.002 \\
\hline Eccentricity & 12.756 & 12.647 & 0.109 \\
\hline Devilation & 9.878 & 9.876 & 0.002 \\
\hline Length & 298.892 & 298.894 & -0.002 \\
\hline The lowest point $\left(X_{0}\right)$ & 136.290 & 136.363 & -0.073 \\
\hline The lowest point $\left(Z_{0}\right)$ & -8.270 & -8.284 & 0.007 \\
\hline
\end{tabular}




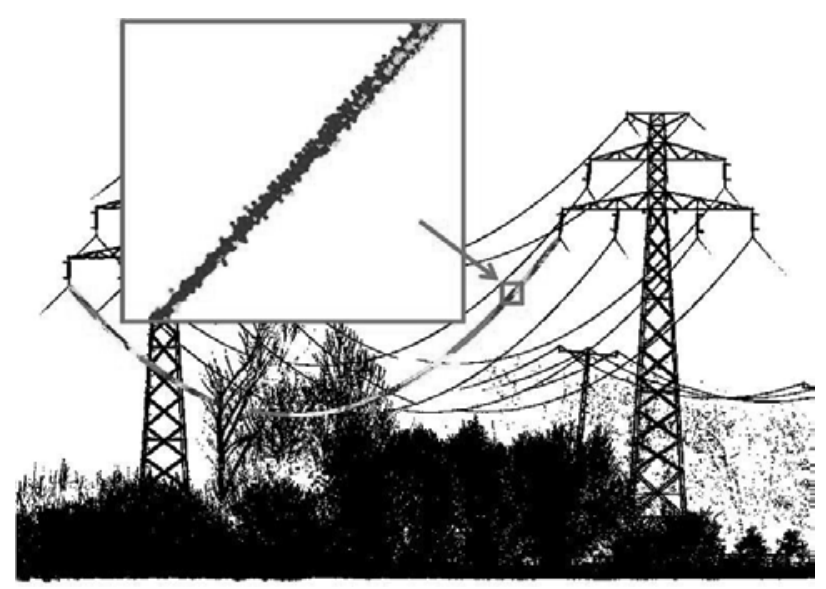

Fig. 4. Visualisation of the point cloud with the analysed cable marked in red colour.

Such a big accumulation of data as used in example presented required the usage of the appropriate computer, due to the fact that either calculations as well as the visualisation required the large computer memory and the good graphics card, but even then it took a long time to receive the results of calculations and to refresh the picture.

Presented in the Paper the example of the analysis was initial and preparatory, moreover it is only one of stages for further processing. Further actions would lead to automation of the calculations' process and the visualisation of results. Algorithms obtained would reprocessed into VisualBasic language (MicroStation Basic) [27], what would allow to use them directly from the MICROSTATION level.

\section{Summary}

The usage of the laser scanning technology in geodesy is not a novelty anymore and become the common measuring technology. Decreasing prices of measuring instruments and growing access to the professional software leads to increasing interest in this measuring technology.

Determining of the overhead power liens' geometry is the issue quite commonly encountered in the geodetic practice. The required accuracy as well as the scope of measurements need to be defined dependent on the aim of measurements and analyses. In some cases, it is not possible to conduct classic measurements due to the terrestrial limitations (lack of appropriate visibility), in the same time usage of the Aerial Photogrammetric Survey or the mobile laser scanning are not economically justifiable. In those cases, the best possible solution is to apply the terrestrial laser scanning method.

It should be emphasized that the methodology of performing TLS measurements and calculating its results was not the subject of this work. Therefore, the results of TLS measurements have been described to a minimum without details. The description of TLS measurements will form the basis of another article

In the Paper, succeeding steps of calculations connected with the analysis of measurements' outcomes and the visualisation of them have been presented. The mathematical relations used to describe the geometry of a hanging cable have been depicted depending on the adopted approximate theoretical function. Calculations and the presentation of results have been illustrated with the example.

Results of approximation for two theoretical curves, those are a catenary and a parabola, have been compared. Obtained results have confirmed that both of those theoretical functions can be applied to calculations.

In the Paper has been used the original software (special made) for the analysis and the visualisation of a overhead power lines geometry. Subsequent steps of the analysis can be automated but the particular caution has to be kept and results obtained have to be verified. The quality and the accuracy of obtained outcomes extensively depends on correctly planned and conducted measurements as well as on correct calculations.

\section{References}

1. M. Antrop, Landscape and Urban Planning, 67, Issues 1-4, 9-26 (2004)

2. M. Hopkowicz, S.M. Rybicki, Geomatics, Landmanagement and Landscape, 1, 19-33 (2014)

3. F. Kiessling, P. Nefzger, J.F. Nolasco, U. Kaintzyk, Overhead Power Lines: Planning, Design, Construction (Springer, 2014)

4. A. Hatibovic, Geomatics, Electrotehnica, Electronica, Automatica, 60, 3, 9-14 (2012)

5. Task Force B2.12.3, Sag-Tension Calculation Methods for Overhead Lines (CIGRE 324 Technical Brochures, 2016)

6. J. Migas, J. Hauschild, Prace geodezyjne przy budowie linii $i$ stacji elektroenergetycznych (ELBUD, Kraków, 1996)

7. D.A. Douglass, R. Thrash, Sag and Tension of Conductor (Taylor and Fransis Group LLC., 2006)

8. A. Wehr, U. Lohr, ISPRS Journal of Photogrammetry and Remote Sensing, 54, 68-82 (1999)

9. P. Amon, U. Riegl, P. Rieger, M. Pfennigbauer, UAV-based laser scanning to meet special challenges in lidar surveying (Geomatics Indaba Proceedings, 2015)

10. L. Matikainen, M. Lehtomäki, E. Ahokas, J. Hyyppä, M. Karjalainen, A. Jaakkola, A. Kukko, T. Heinonen, ISPRS Journal of Photogrammetry and Remote Sensing, 119, 10-31 (2016)

11. S. Kamboj, R. Dahiya, International Journal on Electrical Engineering and Informatics, 3, 3, 268277 (2011)

12. P. Ćwiąkała, Prace Naukowe Instytutu Górnictwa Politechniki Wrocławskiej. Konferencje, 120, 49, 89-97 (2007)

13. J. Kremer, Power Line Mapping: Data Acquisition with A Specialized Multi-Sensor Platform 
(Photogrammetric Week '11, Wichmann/VDE Verlag, 2011)

14. T. Melzer, C. Briese, Extraction and modeling of power lines from ALS point clouds (Proceeding of $28^{\text {th }}$ OAGM Workshop, Austrian Association for Pattern Recognition, Osterreichische Computer Gesellschaft, Hagenberg, Austia, 2004)

15. B. Kwoczyńska, J. Dobek, Journal of Ecological Engineering, 17, 4, 65-74 (2007)

16. P. Axelsson, ISPRS Journal of Photogrammetry and Remote Sensing, 54, 4, 138-147 (1999)

17. B. Song, X. Li, Neurocomputing, 129, 350-361 (2014)

18. G. Yan, C. Li, G. Zhou, W. Zhang, X. Li, IEEE Geoscience and Remote Sensing Letters, 4, 3, 387391 (2007)

19. Y. Jwa, G. Sohn, H. B. Kim, The International Archives of the Photogrammetry, Remote Sensing and Spatial Information Sciences, XXXVIII, Part 3/W8, 105-110 (2009) 